\title{
A Soft Computing System to Perform Face milling Operations
}

\author{
Raquel Redondo ${ }^{1}$, Pedro Santos ${ }^{1}$, Andres Bustillo ${ }^{1}$, Javier Sedano ${ }^{2}$, José Ramón \\ Villar $^{3}$, Maritza Correa ${ }^{4}$, José Ramón Alique ${ }^{4}$, Emilio Corchado ${ }^{1}$ \\ ${ }^{I}$ Department of Civil Engineering, University of Burgos, Burgos, Spain \\ ${ }^{2}$ Department of Electromechanical Engineering. University of Burgos, \\ Burgos, Spain \\ ${ }^{3}$ Department of Computer Science, University of Oviedo, Oviedo, Spain \\ ${ }^{4}$ Department of Industrial Informatic, Instituto de Automática Industrial - \\ Spanish National Research Council, Madrid, Spain \\ emails: rredondo@ubu.es, psgonzalez@ubu.es, abustillo@ubu.es, jsedano@ubu.es, \\ villarjose@uniovi.es, macorrea@iai.csic.es, jralique@,iai.csic.es, escorchado@ubu.es
}

\begin{abstract}
In this paper we present a soft computing system developed to optimize the face milling operation under High Speed conditions in the manufacture of steel components like molds with deep cavities. This applied research presents a multidisciplinary study based on the application of neural projection models in conjunction with identification systems, in order to find the optimal operating conditions in this industrial issue. Sensors on a milling centre capture the data used in this industrial case study defined under the frame of a machine-tool that manufactures industrial tools. The presented model is based on a two-phase application. The first phase uses a neural projection model capable of determine if the data collected is informative enough. The second phase is focus on identifying a model for the face milling process based on low-order models such as Black Box ones. The whole system is capable of approximating the optimal form of the model. Finally, it is shown that the BoxJenkins algorithm, which calculates the function of a linear system from its input and output samples, is the most appropriate model to control such industrial task for the case of steel tools.
\end{abstract}

\section{Introduction}

Soft computing represents a collection or set of computational techniques and intelligent systems principles in machine learning, computer science and some engineering disciplines, which investigate, simulate, and analyze very complex issues and phenomena in order to solve real-world problems. High Speed Machining is nowadays a technology widely used to manufacture many different industrial tools. High Speed Machining, in general, consists on the machining - milling, boring, etcunder cutting conditions where the transfer of generated heat from chip to tool could not take place. 
The industrial expansion of High Speed Machining requires the optimization of its different subprocesses. This optimization depends mainly on the spindle capabilities. The spindle is the element of the machine that provides power and torque to the cutting tool. The subprocesses that requires less power and torque from the machine, like the finishing, are easily optimized, because if the programmed cutting conditions are too high, no damage is expected as for the machine as for the milled piece. Therefore the trial and error method is mainly used in the industry for theses subprocesses. But the subprocesses that require the highest power or torque capabilities usually are not so easily optimizable under industrial conditions. If the programmed cutting conditions are too high, damage could easily be produced to the spindle. The most critical subprocess is the roughing, the first stage of the machining process. During this operation, the highest cutting forces of the whole process will take place. Therefore it is necessary to optimize the cutting conditions to maximise productivity and to avoid damage to the spindle. This difficult balance is especially important during the first task of the roughing operation of deep cavities: the face milling or slotting. This task mills a slot on the metallic piece that will allow the roughing of the deep cavity following a contourning strategy, a softer strategy in terms of cutting forces. Although cutting forces could be calculated [1], the result of this calculation can be only considered as estimation mainly because the chip formation process is still not well known [18]. Then a soft computing model for face milling that could predict the generated cutting forces will contribute to maximise the productivity and the industrial use of High Speed Milling on roughing operations.

In this case we focus on high speed face milling of steel components. Two of the main applications of this technology in this material are the roughing of deep cavities in molds and the machining of slots in machine-tools tables. The soft computing model proposed in this paper is able to predict the cutting forces level for steel face milling depending only on cutting conditions and tool parameters.

\section{An Industrial Process to Perform Face Milling Operations}

Cooperative Maximum-Likelihood Hebbian Learning (CMLHL) [3] is used in this research in order to analyse the internal structure of the data set, which describe the face milling of a steel piece to establish whether it is "sufficiently informative". In the worse case, the experiments have to be performed again in order to collect a proper and informative data set.

CMLHL is a Exploratory Projection Pursuit (EPP) method [2] [4], [5]. In general, EPP provides a linear projection of a data set, but it projects the data onto a set of basic vectors which help reveal the most interesting data structures; interestingness is usually defined in terms of how far removed the distribution is from the Gaussian distribution [6].

One connectionist implementation is Maximum-Likelihood Hebbian Learning (MLHL) [5], [7]. It identifies interestingness by maximising the probability of the residuals under specific probability density functions that are non-Gaussian. An extended version is the CMLHL [3] model, which is based on MLHL [5], [7] but adds 
lateral connections [8], [3] that have been derived from the Rectified Gaussian Distribution [6].

Considering an $\mathrm{N}$-dimensional input vector $(x)$, and an M-dimensional output vector $(y)$, with $W_{i j}$ being the weight (linking input $j$ to output $i$ ), then CMLHL can be expressed [8] as:

1. Feed-forward step:

$$
y_{i}=\sum_{j=1}^{\mathbf{N}} W_{i j} x_{j}, \forall i .
$$

2. Lateral activation passing:

$$
y_{i}(t+1)=\left[y_{i}(t)+\tau(b-A y)\right]^{+} .
$$

3. Feedback step:

$$
e_{j}=x_{j}-\sum_{i=1}^{M} W_{i j} y_{i}, \forall j
$$

4. Weight change:

$$
\Delta W_{i j}=\eta \cdot y_{i} \cdot \operatorname{sign}\left(e_{j}\right)\left|e_{j}\right|^{p-1} .
$$

Where: $\eta$ is the learning rate, $\tau$ is the "strength" of the lateral connections, $b$ the bias parameter, $p$ a parameter related to the energy function [3], [8] and $A$ a symmetric matrix used to modify the response to the data [3]. The effect of this matrix is based on the relation between the distances separating the output neurons.

\subsection{The identification criterion}

The identification criterion evaluates which of the group of candidate models is best adapted to and which best describes the data sets collected in the experiment; i.e., given a model $M\left(\theta_{*}\right)$ its prediction error may be defined by equation (5); and a good model [9] will be that which makes the best predictions, and which produces the smallest errors when compared against the observed data. In other words, for any given data group $Z^{t}$, the ideal model will calculate the prediction error $\varepsilon(t, \theta)$, equation (5), in such a way that for any one $\mathrm{t}=\mathrm{N}$, a particular $\hat{\theta}_{N}$ (estimated parametrical vector) is selected so that the prediction error $\varepsilon\left(t, \hat{\theta}_{N}\right)$ in $\mathrm{t}=1,2,3 \ldots \mathrm{N}$, is made as small as possible.

$$
\varepsilon\left(t, \theta_{*}\right)=y(t)-\hat{y}\left(t \mid \theta_{*}\right) .
$$

The estimated parametrical vector $\hat{\theta}$ that minimizes the error, equation (8), is obtained from the minimization of the error function (6). This is obtained by applying the least-squares criterion for the linear regression, i.e., by applying the quadratic norm $\ell(\varepsilon)=\frac{1}{2} \varepsilon^{2}$, equation (7). 


$$
\begin{gathered}
V_{N}\left(\theta, Z^{N}\right)=\frac{1}{N} \sum_{t=1}^{N} \ell\left(\varepsilon_{F}(t, \theta)\right) . \\
V_{N}\left(\theta, Z^{N}\right)=\frac{1}{N} \sum_{t=1}^{N} \frac{1}{2}(y(t)-\hat{y}(t \mid \theta))^{2} . \\
\hat{\theta}=\hat{\theta}_{N}\left(Z^{N}\right)=\underset{\theta \in D_{M}}{\arg \min } V_{N}\left(\theta, Z^{N}\right) .
\end{gathered}
$$

The methodology of black-box structures has the advantage of only requiring very few explicit assumptions regarding the pattern to be identified, but that in turn makes it difficult to quantify the model that is obtained. The discrete linear models may be represented through the union between a deterministic and a stochastic part, equation (9); the term e(t) (white noise signal) includes the modelling errors and is associated with a series of random variables, of mean null value and variance $\lambda$.

$$
y(t)=G\left(q^{-1}\right) u(t)+H\left(q^{-1}\right) e(t) .
$$

The structure of a black-box model depends on the way in which the noise is modelled $H\left(q^{-1}\right)$; thus, if this value is 1 , then the OE (Output Error) model is applicable; whereas, if it is different from zero a great range of models may be applicable; one of the most common being the BJ (Box Jenkins) algorithm. This structure may be represented in the form of a general model, where $B\left(q^{-1}\right)$ is a polynomial of grade $\mathrm{n}_{\mathrm{b}}$, which can incorporate pure delay $\mathrm{n}_{\mathrm{k}}$ in the inputs, and $A\left(q^{-1}\right), C\left(q^{-1}\right), D\left(q^{-1}\right)$ and $F\left(q^{-1}\right)$ are autoregressive polynomials ordered as $\mathrm{n}_{\mathrm{a}}$, $\mathrm{n}_{\mathrm{c}}, \mathrm{n}_{\mathrm{d}}, \mathrm{n}_{\mathrm{f}}$, respectively (10). Likewise, it is possible to use a predictor expression, for the on-step prediction ahead of the output $\hat{y}(t \mid \theta)$ (11). In Table 1, the generalized polynomial expressions are presented, as well as those that represent the polynomials used in the case of each particular model.

$$
\begin{gathered}
A\left(q^{-1}\right) y(t)=q^{-n_{k}} \frac{B\left(q^{-1}\right)}{F\left(q^{-1}\right)} u(t)+\frac{C\left(q^{-1}\right)}{D\left(q^{-1}\right)} e(t) \\
\hat{y}(t \mid \theta)=\frac{D\left(q^{-1}\right) B\left(q^{-1}\right)}{C\left(q^{-1}\right) F\left(q^{-1}\right)} u(t)+\left[1-\frac{D\left(q^{-1}\right) A\left(q^{-1}\right)}{C\left(q^{-1}\right)}\right] y(t)
\end{gathered}
$$

Table 1. Black-box model structures

\begin{tabular}{|l|l|l|}
\hline \multicolumn{1}{|c|}{ Polynomials in (10) } & $\begin{array}{c}\text { Polynomials used } \\
\text { in (10) }\end{array}$ & $\begin{array}{c}\text { Name of model } \\
\text { structure }\end{array}$ \\
\hline$A\left(q^{-1}\right)=1+a_{1}\left(q^{-1}\right)+a_{2}\left(q^{-2}\right)+\cdots+a_{n_{a}}\left(q^{-n_{a}}\right)$ & & \\
$B\left(q^{-1}\right)=b_{1}\left(q^{-1}\right)+b_{2}\left(q^{-2}\right)+\cdots+b_{n_{b}}\left(q^{-n_{b}}\right)$ & $\mathrm{B}$ & FIR \\
$C\left(q^{-1}\right)=1+c_{1}\left(q^{-1}\right)+c_{2}\left(q^{-2}\right)+\cdots+c_{n_{c}}\left(q^{-n_{c}}\right)$ & ABC & ARX \\
$D\left(q^{-1}\right)=1+d_{1}\left(q^{-1}\right)+d_{2}\left(q^{-2}\right)+\cdots+d_{n_{d}}\left(q^{-n_{d}}\right)$ & AC & ARMAX \\
$F\left(q^{-1}\right)=1+f_{1}\left(q^{-1}\right)+f_{2}\left(q^{-2}\right)+\cdots+f_{n_{f}}\left(q^{-n_{f}}\right)$ & BF & BECD \\
& & BJ \\
\hline
\end{tabular}


Procedure for Modelling the Face Milling Process. The identification procedure used to identify a parameterized model $\mathrm{M}$, which will eventually be selected as the best from among those that modelled the face milling characteristics on the basis of the variable measurements, is carried out in accordance with two fundamental patterns: a first pre-analytical and then an analytical stage that assists with the determination of the parameters in the identification process and the model estimation. The pre-analysis test is run to establish the identification techniques [9], [10], [11], [12], [13], [14], the selection of the model structure and its order estimation [15], [16], the identification criterion and search methods that minimize it and the specific parametrical selection for each type of model structure.

A second validation stage ensures that the selected model meets the necessary conditions for estimation and prediction. Three tests were performed to validate the model: residual analysis $\varepsilon(t, \hat{\theta}(t))$, by means of a correlation test between inputs, residuals and their combinations; final prediction error (FPE) estimate, as explained by Akaike [17]; and the graphical comparison between desired outputs and the outcome of the models through simulation one (or k) steps before.

\section{$3 \quad$ Modelling the Face milling of Steel: An Industrial Task}

To obtain the experimental data required to develop and validate the modelling procedure, different face milling tests were performed. The experimental set-up and procedure were described in detail beforehand [19]. A machining centre Kondia HS1000 equipped with a Siemens 840D open-architecture CNC was used. The blank material used for the tests was a $180 \mathrm{~mm}$ profile of F114 steel. The cutting tools were Karnash end-mill tools model 30.6472 with two flutes and model 30.6455 with six flutes. For the tests, each tool (diameters 6, 8, 10 and $12 \mathrm{~mm}$ ) mechanized slots each one with constant spindle speed maintaining constant feed rate and depth of cut. For all the tests, new tools and combinations of parameters free of chatter were used. A data set of 250 records was obtained. Each record includes the information on the following six variables: tool diameter, tool number of flutes, axial depth of cut, machine feed rate, spindle speed and tangential cutting force. To measure the tangential cutting force a multi-component dynamometer with an upper plate was used. Since the data were thought to be used with Bayesian network algorithms [19], the variables were discretized. The selected discretization algorithm and the possible values that each variable could take are shown in Table 2. 
Table 2. Variables, units, values and discretization algorithms used during the experiments. All values are common to this face milling process. Output $y(t)$, Input $u(t)$.

\begin{tabular}{|l|c|c|}
\hline \multicolumn{1}{|c|}{ Variable (Units) } & Possible values & Discretization algorithm \\
\hline Tangential cutting force $(\mathrm{N}), \mathrm{y}_{1}(\mathrm{t})$ & $0,1,2,3$ & Fuzzy K-means algorithm [20] \\
\hline Tool diameter $(\mathrm{mm}), \mathrm{u}_{1}(\mathrm{t})$ & $6,8,10,12$ & Already discrete \\
\hline Tool number of flutes, $\mathrm{u}_{2}(\mathrm{t})$ & 2,6 & Already discrete \\
\hline Axial depth of cut $(\mathrm{mm}), \mathrm{u}_{3}(\mathrm{t})$ & $2,4,6,8,10,12$ & Already discrete \\
\hline Machine feed rate $(\mathrm{mm} / \mathrm{min}), \mathrm{u}_{4}(\mathrm{t})$ & $0,1,2,3$ & K-means algorithm [21] \\
\hline Spindle speed $\left(\mathrm{min}^{-1}\right), \mathrm{u}_{5}(\mathrm{t})$. & $0,1,2,3$ & Fuzzy K-means algorithm [20] \\
\hline
\end{tabular}

\subsection{Application of the two phases of the modelling system}

The study has been organized into two phases or steps.

Step 1: Analysis of the internal structure of the data set based on the application of several unsupervised connectionist models.

Step 2: Application of several identification models in order to find the one that best defines the dynamic of a face milling process.

Step 1. Figure 1.b shows the results obtained by means of CMLHL projections. This model is able to identify four different clusters order mainly by Feed rate. After studying each cluster it can be noted a second classification based on the tangential cutting force and spindle speed as it is shown in Fig. 1.b. All this indicates that the data analysed is sufficiently informative.

We have also applied PCA. Both methods have identified a clear internal structure based on an initial classification, but CMLHL (Fig. 1.b) provides a sparser representation than PCA (Fig. 1.a)

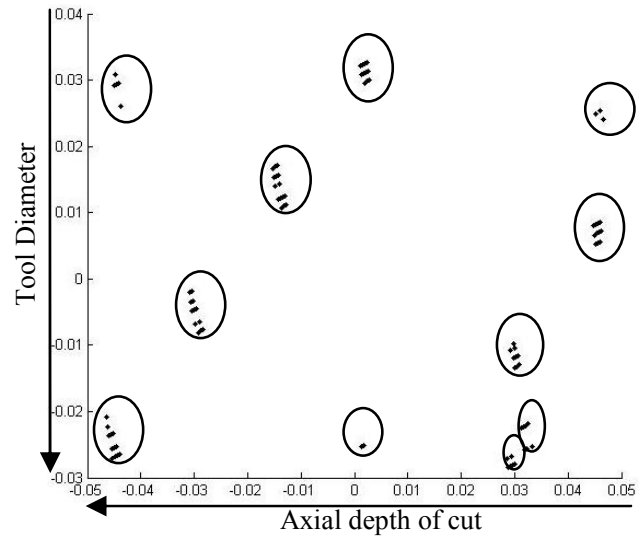

Fig 1.a Projection of PCA

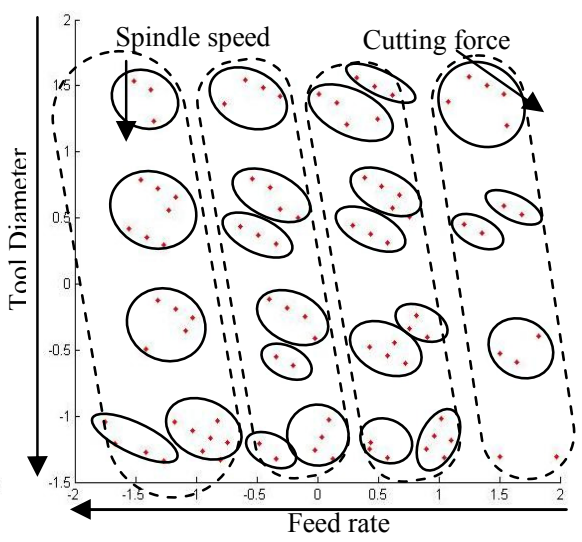

Fig 1.b Projection of CMLHL (Steps= 1000000, $\eta=0.01, p=0.5$ and $\tau=0.1$ ) 
Step 2. Modelling the face milling process. Fig. 2, shows the results of output $\mathrm{y}_{1}(\mathrm{t})$, tangential cutting force $(\mathrm{N})$, for the different models. Fig. 2 shows the graphic representations of the results, for OE y BJ models, in relation to the polynomial order and the delay in the inputs; various delays for all inputs and various polynomial orders $\left[n_{b 1} n_{b 2} n_{b 3} n_{b 4} n_{b 5} n_{c} n_{d} n_{f} n_{k 1} n_{k 2} n_{k 3} n_{k 4} n_{k 5}\right.$ ] were considered to arrive at the highest degree of precision, in accordance with the structure of the models that have been used; see Table 1. In Fig. 2, the X-axis shows the number of samples used in the validation of the model (50 samples in intervals of $0,1 \mathrm{~s}$ ), while the $\mathrm{Y}$-axis represents the normalized output variable, which is the tangential cutting force.

Table 3 shows a comparison of the qualities of estimation and prediction of the best models obtained, as a function of the model, the estimation method, and the indexes, which are defined as follows:

- The percentage representation of the estimated model (expressed as so many percent "\%") in relation to the true system: the numeric value of the normalized mean error that is computed with one-step prediction (FIT1), with ten-step prediction (FIT10), or by means of simulation (FIT). Also shown are the graphical representations of true system output and both the one-step prediction $\hat{y}_{1}(t \mid m)$, the ten-step prediction $\hat{y}_{10}(t \mid m)$, and the model simulation $\hat{y}_{\infty}(t \mid m)$.

- The loss or the error function $(\mathrm{V})$ : the numeric value of the mean square error that is calculated from the estimation data set.

- The generalization error value (NSSE): the numeric value of the mean square error that is calculated from the validation data set.

- The average generalization error value (FPE): This is the numeric value of the FPE criterion that is calculated from the estimation data set.
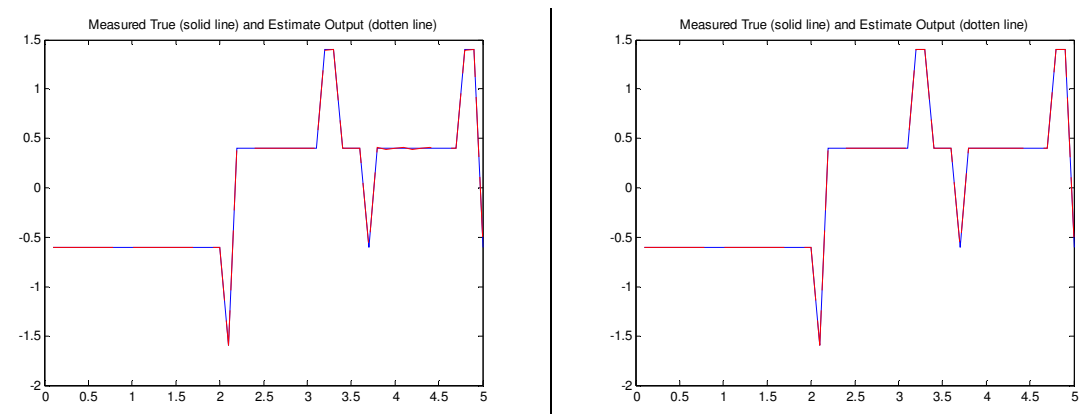

Fig.2. Output response of two different models: the OE -left column- and BJ -right columnmethods. The real measure (solid line) is graphically presented with the simulated output and with one step ahead prediction (dashed line). The order of the structure of the model are $n_{b l}=1$, $n_{b 2}=1, n_{b 3}=3, n_{b 4}=1, n_{b 5}=1, n_{c}=2, n_{d}=1, n_{f}=8, n_{k 1}=1, n_{k 2}=9, n_{k 3}=15, n_{k 4}=10, n_{k 5}=1 .\left[\begin{array}{llllll}1 & 1 & 3 & 1 & 1 & 2\end{array}\right.$ $\left.\begin{array}{lllllll}1 & 8 & 1 & 9 & 15 & 10 & 1\end{array}\right]$, for tangential cutting force $\mathrm{y}_{1}(\mathrm{t})$, according to the model type.

From the graphical representation (Fig.2) it can be concluded that the BJ model is capable of simulating and predicting the behaviour of the tangential cutting force $(\mathrm{N})$ $\left(\mathrm{y}_{1}(\mathrm{t})\right)$ during the face milling process as it meets the indicators and it is capable of 
modelling more than $99 \%$ of the true measurements. The tests were performed using Matlab and the System Identification Toolbox. Table 4 shows the final BJ model.

Table 3. Indicator values for several proposed models of tangential cutting force.

\begin{tabular}{|c|c|}
\hline Model & Indexes \\
\hline $\begin{array}{l}\text { Black-box OE model with } n_{b 1}=1, n_{b 2}=1, n_{b 3}=3, n_{b 4}=1, n_{b 5}=1 \text {, } \\
n_{f}=8, n_{k 1}=1, n_{k 2}=9, n_{k 3}=15, n_{k 4}=10, n_{k 5}=1 . \text { The model is } \\
\text { estimated using the prediction error method, the degree of the } \\
\text { model selection is carried out from the best AIC criterion (the } \\
\text { structure that minimizes AIC). }\end{array}$ & $\begin{array}{l}\text { FIT:99.48\%, FIT1:99.48\% } \\
\text { FIT10:99.48\%, V: } 0.482 \\
\text { FPE:0.936, NSSE: } 1.21 \mathrm{e}-5\end{array}$ \\
\hline $\begin{array}{l}\text { Black-box BJ model with } n_{b 1}=1, n_{b 2}=1, n_{b 3}=3, n_{b 4}=1, n_{b 5}=1 \text {, } \\
n_{c}=2, n_{d}=1, n_{f}=8, n_{k 1}=1, n_{k 2}=9, n_{k 3}=12, n_{k 4}=10, n_{k 5}=1 \text {. The } \\
\text { model is estimated using the prediction error method, the } \\
\text { degree of the model selection is carried out with the best AIC } \\
\text { criterion (the structure that minimizes AIC). }\end{array}$ & $\begin{array}{l}\text { FIT:93.1\%, FIT1:87.25\% } \\
\text { FIT10:93.09\%, V: } 0.313 \\
\text { FPE:0.90, NSSE:0.0071 }\end{array}$ \\
\hline $\begin{array}{l}\text { Black-box BJ model with The model is estimated using the } \\
\text { prediction error method, the degree of the model selection is } \\
\text { carried out with } n_{b 1}=1, n_{b 2}=1, n_{b 3}=3, n_{b 4}=1, n_{b 5}=1, n_{c}=2 \text {, } \\
n_{d}=1, n_{f}=8, n_{k 1}=1, n_{k 2}=9, n_{k 3}=15, n_{k 4}=10, n_{k 5}=1 \text {. the best AIC } \\
\text { criterion (the structure that minimizes AIC). }\end{array}$ & $\begin{array}{l}\text { FIT: } 100 \%, \text { FIT1: } 100 \% \\
\text { FIT10:100\%, V: } 0.258 \\
\text { FPE: } 0.775 \text {, NSSE: } 1.48 \mathrm{e}-12\end{array}$ \\
\hline
\end{tabular}

Table 4. Function and parameters that represent the behaviour of face milling process for the tangential cutting force. The degree of the BJ model polynomials are $n_{b 1}=1, n_{b 2}=1$, $n_{b 3}=3, n_{b 4}=1, n_{b 5}=1, n_{c}=2, n_{d}=1, n_{f}=8, n_{k 1}=1, n_{k 2}=9, n_{k 3}=15, n_{k 4}=10, n_{k 5}=1$. [ $\begin{array}{lllllllll}1 & 1 & 3 & 1 & 1 & 2 & 1 & 8 & 1\end{array}$ $915101]$.

Parameters and polynomials.

\begin{tabular}{|c|c|}
\hline$B 1(q)=0.003639 q^{-1}$ & $B 2(q)=-0.09618 q^{\wedge^{-9}}$ \\
\hline $\begin{array}{l}B 3(q)=-0.02963 q^{-15}-0.1024 q^{-16}- \\
0.01665 q^{-17}\end{array}$ & $B 4(q)=-0.02778 q^{-10}$ \\
\hline$B 5(q)=-0.0631 q^{-1}$ & $C(q)=1+0.06222 q^{-1}+0.003241 q^{-2}$ \\
\hline$D(q)=1-0.7596 q^{-1}$ & $\begin{array}{l}F 1(q)=1+0.02932 q^{-1}-0.1422 q^{-2}+1.13 \\
q^{-3}-0.3719 q^{-4}-0.07694 q^{-5}+0.2387 q^{-6}- \\
0.2382 q^{-7}+0.2322 q^{-8}\end{array}$ \\
\hline $\begin{array}{l}F 2(q)=1-1.18 q^{-1}+0.888 q^{-2}-0.260 \\
q^{-3}-0.4723 q^{-4}+0.8265 q^{-5}-0.5742 q^{-} \\
6+0.3817 q^{-7}-0.4978 q^{-8}\end{array}$ & $\begin{array}{l}F 3(q)=1+0.261 q^{-1}+0.799 q^{-2}+0.1734 \\
q^{-3}+1.093 q^{-4}+0.04379 q^{-5}+0.6479 q^{-6}+ \\
0.1822 q^{-7}+0.4301 q^{-8}\end{array}$ \\
\hline $\begin{array}{l}F 4(q)=1-0.2047 q^{-1}+0.5632 q^{-2}- \\
0.4963 q^{-3}+1.041 q^{-4}-0.07187 q^{-5}+ \\
0.2924 q^{-6}-0.2778 q^{-7}+0.1622 q^{-8}\end{array}$ & $\begin{array}{l}F 5(q)=1-0.4916 q^{-1}-0.01854 q^{-2}+ \\
0.6543 q^{-3}-0.5834 q^{-4}+0.4833 q^{-5}-0.2851 \\
q^{-6}-0.1829 q^{-7}+0.4553 q^{-8} \\
e(t) \text { is white noise signal whit variance } 0.52\end{array}$ \\
\hline
\end{tabular}

The obtained model can be used not only to predict the tangential cutting force but also to determine the optimal conditions to minimize it. Considering that the model is a polynomial model, if all but one input variables are fixed then the remaining variable could be calculated and fixed in order to minimize the tangential cutting 
force. This procedure could be used to assure that the axial cutting force will never be higher than the damage threshold of the spindle.

\section{Conclusions and Futures lines of Work}

This paper presents a study to identify the most appropriate modelling system to perform face milling operations. Different models were analysed to achieve the best practical solution to this industrial problem. The study shows that the Box-Jenkins algorithm is best adapted to this case, as the noise model is better, been lower than the other model (OE).

Future work will be focus on the generation of the system inputs in a way that they minimize the output error, in a manner that the customer has access to a graphical representation allowing him to choose the inputs values in order to obtain the best response. Also we will extend this study to other kind of materials of industrial interest, such as aeronautical aluminium. We are also working on the application of this model to the optimization of different but similar industrial problems, like sloting by turning of steel components for wind generators, like seals groves of gearboxes covers.

\section{Acknowledgments}

This research has been partially supported through project BU006A08 of Junta de Castilla y León, through projects CIT-020000-2008-2 and TIN2007-62626 of Spanish Ministry of Science and Innovation and the MOCAVE Project under Grant DPI200612736-C02-01. The authors would also like to thank the manufacturer of components for vehicle interiors, Grupo Antolin Ingeniería, S.A. in the framework of the project MAGNO 2008 - 1028.- CENIT Project funded by the Spanish Ministry of Science and Innovation.

\section{References}

1. Altintas, Y.: Manufacturing Automation: Metal Cutting Mechanics, Machine Tool Vibrations, and CNC Design, Cambridge University Press (2000)

2. Diaconis, P. and Freedman, D.: Asymptotics of Graphical Projections, The Annals of Statistics, 12(3), pp. 793-815, (1984).

3. Corchado, E. and Fyfe, C.: Connectionist Techniques for the Identification and Suppression of Interfering Underlying Factors. Int. Journal of Pattern Recognition and Artificial Intelligence, 17(8) (2003), pp. 1447-1466.

4. Friedman, J.H. and Tukey, J.W.: Projection Pursuit Algorithm for Exploratory DataAnalysis, IEEE Transactions on Computers, 23(9), pp. 881-890, (1974). 
5. Corchado, E., MacDonald, D. and Fyfe, C: Maximum and Minimum Likelihood Hebbian Learning for Exploratory Projection Pursuit. Data Mining and Knowledge Discovery, 8(3), pp. 203-225, (2004).

6. Seung, H.S., Socci, N.D. and Lee, D.: The Rectified Gaussian Distribution, Advances in Neural Information Processing Systems, 10, pp. 350-356, (1998).

7. Fyfe, C. and Corchado, E.: Maximum Likelihood Hebbian Rules, Proc. of the 10th European Symposium on Artificial Neural Networks (ESANN 2002), pp. 143-148, (2002).

8. Corchado, E., Han, Y. and Fyfe, C.: Structuring Global Responses of Local Filters Using Lateral Connections, Journal of Experimental \& Theoretical Artificial Intelligence, 15(4), pp. 473-487, (2003).

9. Ljung, L.: System Identification, Theory for the User, Prentice-Hall, (1999).

10. Nögaard, M., Ravn, O., Poulsen, N. K. and Hansen, L. K.:Neural Networks for Modelling and Control of Dynamic Systems, Springer-Verlag, London, U.K., (2000).

11. Söderström, T. and Stoica, P. : System identification, Prentice Hall, (1989).

12. Nelles, O.: Nonlinear System Identification, From Classical Approaches to Neural Networks and Fuzzy Models, Springer, (2001).

13. Haber, R. and Keviczky, L.: Nonlinear System Identification, Input-Output Modelling Approach, Part. 2: Nonlinear System structure Identification, Kluwer Academic Publishers, (1999).

14. Haber, R. and Keviczky, L.: Nonlinear System Identification, Input-Output Modelling Approach, Part 1: Nonlinear System Parameter Estimation, Kluwer Academic Publishers, (1999).

15. Stoica, P. and Söderström, T.: A useful parametrization for optimal experimental design, In IEEE Trans. Automatic. Control, AC-27,(1982).

16. He, X. and Asada, H.: A new method for identifying orders of input-output models for nonlinear dynamic systems, In Proc. Of the American Control Conf., S. F., pp. 2520-2523, California, (1993).

17. Akaike, H.: Fitting autoregressive models for prediction, Ann. Inst. Stat. Math., 20, pp. 425-439, (1969).

18. Behrens A., Westhoff B., Fundamental aspects of investigating the HSC-Chip formation process by FEM. Scientific Fundamentals of HSC, Carl Hanser Verlag, (2001)

19. Correa, M., Bielza, C., Ramirez, M. de J. and Alique, J. R. A Bayesian network model for surface roughness prediction in the machining process. International Journal of Systems Science, 39(12), pp. $1181-1192$ (2008).

20. Duda, R.O., Hart, P.E., and Stork, D.G., Pattern Classification, NY: Wiley (2001).

21. MacQueen, J. B., Some methods for classification and analysis of multivariate observations. $5^{\text {th }}$ Berkeley Symposium on Mathematical Statistics and Probability (Vol. 1), pp. 281-297 (1967). 